\title{
ANÁLISIS EXERGÉTICO DE UNA PLANTA DE COGENERACIÓN OPERANDO BAJO CICLO COMBINADO
}

\section{Exergy analysis of cogeneration plant operating under combined cycle}

\author{
Hernán Darío Patiño Duque ${ }^{1}$, Brayan Darío Rosero Coral ${ }^{1}$ \\ ${ }^{1}$ Universidad Tecnológica de Pereira -UTP, Facultad de Ingeniería Mecánica, Grupo inv. Genergética, \\ Colombia. E-mail: hedapatino@utp.edu.co, bdrosero@utp.edu.co
}

(Recibido Julio 27 de 2016 y aceptado Octubre 21 de 2016)

\begin{abstract}
Resumen
En la actualidad, hay diversas formas para generar energía eléctrica; una de ellas consiste en aprovechar la cantidad de energía liberada al quemar un combustible y efectuar una serie de procesos de conversión de energía mediante un ciclo termodinámico. Para hacer estos procesos más eficientes, existen técnicas que permiten analizar en qué equipos de la planta se está desaprovechando energía; una de estas técnicas es el análisis exergético, que consiste en la aplicación simultanea de la primera y segunda ley de la termodinámica. En el presente trabajo se realiza el análisis exergético a una planta de generación de electricidad y calor a partir de la producción de vapor operando bajo un ciclo combinado. Inicialmente se definen conceptos importantes para realizar el análisis. Después de aplicar la primera y segunda ley de la termodinámica los resultados indican que la eficiencia exergética de la planta de ciclo combinado es $53 \%$ y se encuentra que la cámara de combustión es el componente del sistema que más exergía destruye y que las bombas son los dispositivos donde hay menor pérdida de energía útil.
\end{abstract}

Palabras clave: Balance exergético, ciclo combinado, cogeneración, energía, irreversibilidad.

\begin{abstract}
Nowadays, there are several ways to generate electricity; one of them is to harness the amount of energy released after burning a fuel and carrying out a series of processes to convert energy through a thermodynamic cycle. To make these processes more efficient, there are techniques that allows to analyze which plant devices are wasting energy; one of these techniques is exergy analysis which consists on a simultaneous application of the first and second law of thermodynamics. This paper shows the exergy analysis in a plant of electricity and heat generation from the steam production operating under a combined cycle. Initially, important concepts are defined to perform exergy analysis. After applying the first and second law of thermodynamics, the results indicate that the exergy efficiency of the plant of combined cycle is $53 \%$ and the combustion chamber is the device that destroyed more exergy in the system and the pumps are devices where occur less destruction of exergy.
\end{abstract}

Key words: Exergy balance, combined cycle, cogeneration, energy, irreversibility.

\section{INTRODUCCIÓN}

La energía eléctrica tiene un papel clave en el desarrollo económico y social de un país, esto ha hecho que compañías empiecen a generar electricidad por medio de centrales termoeléctricas de ciclo combinado [1]. Las plantas de ciclo combinado acoplan el ciclo Brayton con el ciclo Rankine [2]. La idea básica consiste en utilizar la energía contenida en los gases de escape de la cámara de combustión, en un proceso de vapor conectado por medio de una caldera de recuperación aguas abajo de la turbina de gas [3]. En una central de ciclo combinado la caldera de recuperación HRSG (heat recovery steam generator) representa el elemento de la interfaz entre la turbina de gas y el ciclo de vapor. Aquí, los gases de escape de la turbina de gas se enfrían y el calor recuperado 


\begin{tabular}{|c|c|c|c|}
\hline \multicolumn{2}{|c|}{ Nomenclatura } & \multicolumn{2}{|c|}{ Subíndices } \\
\hline$b$ & exergía especifica [kJ/kg] & $a$ & aire \\
\hline$B$ & Flujo de exergía [kW] & $\mathrm{e}$ & entrada \\
\hline$B_{D}$ & flujo de exergía destruida [kW] & $f$ & combustible \\
\hline$C_{p}$ & calor especifico a presión constante [kJ/kg K] & 0 & estado muerto \\
\hline$h$ & entalpia especifica [KJ/kg] & v & vapor \\
\hline$k$ & constante adiabática [-] & s & salida \\
\hline$\dot{m}$ & flujo másico [kg/s] & & \\
\hline$P C I$ & poder calorífico inferior [KJ/kg] & \multirow{2}{*}{\multicolumn{2}{|c|}{ Abreviaciones }} \\
\hline$\phi$ & flujo de calor [KW] & & \\
\hline$r_{p}$ & relación de compresión [-] & C & compresor \\
\hline$s$ & entropía especifica [KJ/kg K] & $\mathrm{CC}$ & cámara de combustión \\
\hline$T$ & temperatura $\left[{ }^{\circ} \mathrm{C}\right]$ & Cond & condensador \\
\hline$W$ & Potencia & HRSG & caldera de recuperación \\
\hline & & $\mathrm{IA}$ & intercambiador abierto \\
\hline \multirow[t]{2}{*}{ Letra griega } & & IC & intercambiador cerrado \\
\hline & & TG & turbina de gas \\
\hline$\eta$ & eficiencia energética & $\mathrm{P}$ & Proceso industrial \\
\hline \multirow[t]{2}{*}{$\bar{\varepsilon}$} & eficiencia exergética & TVAP & turbina de vapor de alta presión \\
\hline & & TVBP & turbina de vapor de baja presión \\
\hline
\end{tabular}

Se utiliza para generar vapor. Con el fin de proporcionar una mejor recuperación de calor en la HRSG, se suele utilizar más de un nivel de presión [4]. Las plantas que operan bajo ciclo combinado presentan ventajas respecto a las de ciclo Rankine y Brayton independientes; algunas de estas ventajas son la relación costos beneficios y el bajo impacto ambiental; según [5] una central de ciclo combinado con gas natural requiere una inversión de unos $500 € / \mathrm{kW}$, y alcanza un rendimiento bruto cercano al $59 \%$ a plena carga, una central térmica de carbón, incluso las dotadas con calderas supercríticas de última generación y sistemas de depuración de gases de combustión, tienen un rendimiento del orden del
$45 \%$ con costos de inversión en el entorno de los 1000 $€ /$ kW.

Las configuraciones de las plantas de ciclo combinado han venido cambiando, debido a las necesidades de las industrias, es aquí donde sale el término cogeneración [6]. La cogeneración es la producción simultánea de energía eléctrica o mecánica y energía térmica útil a partir de un único flujo de energía (combustible) como el petróleo, carbón, gas natural o licuado, biomasa [7]. Resulta favorable hablar de cogeneración donde hay demanda de energía térmica, como el caso de los procesos industriales. 
Con el fin de mejorar la eficiencia y reducir el desperdicio de energía en una planta, se han planteado diferentes metodologías de análisis, una de éstas es el análisis exergético [8]. Ésta técnica se plantea con base a la segunda ley de la termodinámica y es considerada como una herramienta poderosa en el análisis de sistemas térmicos, para identificar los lugares y las magnitudes de las irreversibilidades; cabe resaltar que algunas de las irreversibilidades que se encuentran después de hacer un análisis exergético no pueden ser eliminadas, pero si se pueden mejorar, ya sea rediseñando el equipo o el proceso donde se presenten $[9,17]$.

Para la evaluación del desempeño termodinámico de plantas de ciclo combinado, muchos investigadores han realizado diferentes métodos de análisis. Además del análisis de primera ley de la termodinámica, aplicaron la segunda ley para calcular el flujo de exergía destruida y la eficiencia exergética de cada componente de la planta.

"Según [10]", se realizó el análisis exergético a la planta de ciclo combinado ATAER, localizada en la zona industrial Izmir Atatürk-Turquia; esta planta entró en funcionamiento en Octubre de 1998 con una sola turbina de gas, en el 2001 se le adicionó una turbina de vapor y empezó a operar como ciclo combinado. En el año 2009 se le instaló una caldera de recuperación y otra turbina de gas. Ersayin obtuvo como resultados: eficiencia energética del $56 \%$ y rendimiento exergético del 50.04\%. En la referencia [11], aplicó un análisis exergético convencional y avanzado a una planta de ciclo combinado con recalentamiento. Con el análisis convencional identificó las irreversibilidades en el sistema y con el análisis avanzado encontró las exergías endógenas y exógenas destruidas en cada componente de la planta. [1] propuso la evaluación energética y exergética de una planta de cogeneración hibrida con ciclo Rankine regenerativo. [12] investigó las técnicas para reducir las irreversibilidades de las calderas de recuperación (HRSG) de los ciclos combinados, también modeló y analizó métodos modernos de enfriamiento para ciclos combinados comerciales. De acuerdo al estudio, al reducir las diferencias de temperaturas del pinch point y reducir las tasas de flujo de masa de vapor se reducen las irreversibilidades. En la referencia [13], muestra los resultados de un análisis exergoeconómico a un sistema de cogeneración con turbina de gas y describe la viabilidad de cogeneración en plantas de ciclo combinado. [14] expone el análisis exergético de una central de ciclo combinado con interenfriamiento en la combustión de la turbina; el análisis muestra que la eficiencia en el ciclo con interenfriamiento aumenta en un $3.13 \%$, además la exergía destruida en todos los componentes, baja $2 \%$.

Los estudios en [15], muestra la evaluación del rendimiento de una instalación de generación de electricidad situada en Turquía con un análisis exergético avanzado; esta planta opera bajo ciclo combinado, usa como combustible gas natural y el sistema de conversión de energía consiste de un compresor, una cámara de combustión, una turbina de gas, la caldera de recuperación, turbinas de vapor de alta, baja presión y un condensador.

A diferencia de la literatura donde no se tiene en forma simultanea recalentamiento, cogeneración y envió del agua de alimentación hacia un intercambiador de calor tipo abierto, el presente trabajo pretende evaluar las condiciones de operación asumidas y la configuración de los elementos en la planta de ciclo combinado propuesta en la Figura 1 por medio de un análisis exergético convencional, donde se utiliza esta metodología para identificar los lugares donde se presentan las mayores pérdidas de exergía e ineficiencias, esto con el cálculo del valor de la eficiencia exergética de la planta y la exergía destruida de cada componente.

\section{DESCRIPCIÓN DEL SISTEMA}

Tal como se muestra en la Figura 1, la planta de cogeneración está conformada principalmente por una planta de turbina de gas, donde el combustible utilizado es gas natural, una caldera de recuperación HRSG, una turbina de vapor de alta y una de baja presión, un intercambiador de calor tipo cerrado y uno tipo abierto, a ésta se le puede denominar como planta de cogeneración debido a que produce y suministra energía eléctrica y vapor para un proceso industrial.

\subsection{Supuestos}

* La configuración de éste sistema produce $300 \mathrm{~kg} / \mathrm{s}$ de aire y $31,58 \mathrm{~kg} / \mathrm{s}$ de vapor. La temperatura máxima del ciclo de gas es de $1247^{\circ} \mathrm{C}$.

* En una planta térmica las condiciones ambientales dependen de la ubicación geográfica. Las condiciones

Rev. I²+D. Vol.17 (1). Enero-Junio. 2017, pp. 49-58. ISSN 1900-771X e-ISSN 2422-4324 
de referencia ambientales consideradas en este trabajo son $25^{\circ} \mathrm{C}$ y $101,325 \mathrm{kPa}$, las cuales son acordes a las condiciones de Colombia.

* La temperatura del vapor sobrecalentado en la salida de la caldera de recuperación es de $591,9{ }^{\circ} \mathrm{C}$ con una presión de $6 \mathrm{MPa}$.

* La planta cuenta con un recalentamiento y posteriormente el vapor se expande en una turbina de vapor hasta la presión del proceso industrial que es de $520 \mathrm{kPa}$. La presión de condensación es de $10 \mathrm{kPa}$. Los valores nominales de operación (Presión, temperatura, eficiencias isentrópica de turbinas y bombas, etc...) se muestran en las Tablas 1 y 2.

Tabla 1. Eficiencia isentrópica de los componentes

\begin{tabular}{lc}
\hline Componente & Eficiencia isentrópica \\
\hline C & $84 \%$ \\
CC & $85 \%$ \\
Bombas & $70 \%$ \\
TG & $86 \%$ \\
TVAP, TVBP & $86 \%$ \\
\hline
\end{tabular}

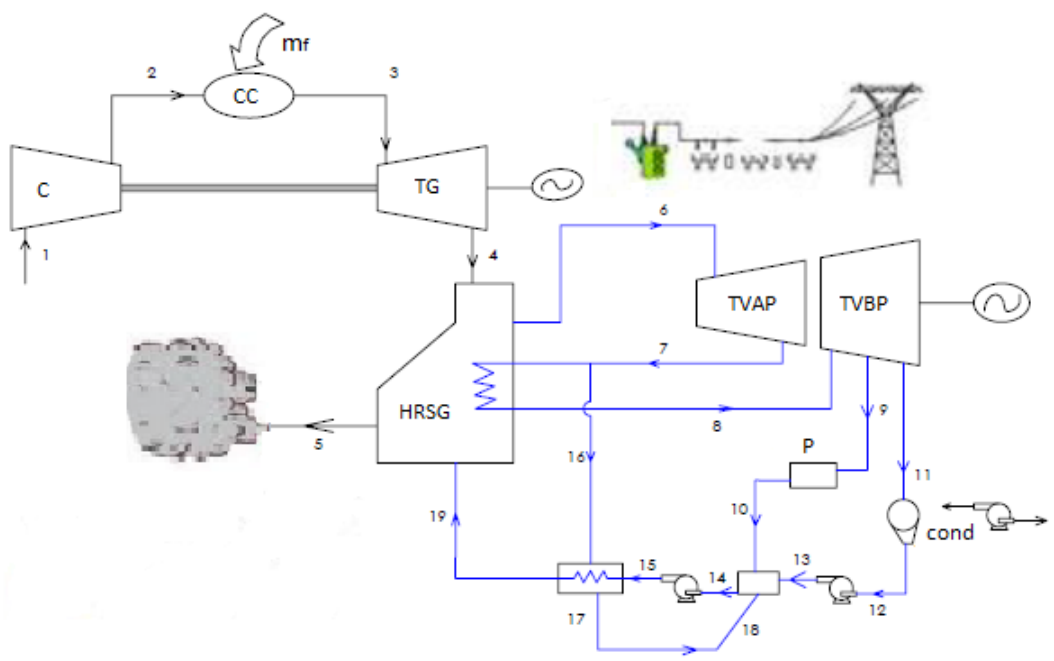

Figura 1. Diagrama de flujo, planta de ciclo combinado.

Tabla 2. Condiciones de operación de la planta propuesta

\begin{tabular}{lc}
\hline Condiciones de operación & Valor \\
\hline Flujo másico de aire & $300(\mathrm{~kg} / \mathrm{s})$ \\
Flujo másico de vapor, entrada TV de alta presión & $31,58(\mathrm{~kg} / \mathrm{s})$ \\
Temp máxima del ciclo de gas & $1247\left({ }^{\circ} \mathrm{C}\right)$ \\
Presión del vapor en la caldera de recuperación & $6000(\mathrm{kPa})$ \\
Temp del vapor en la caldera de recuperación & $591,9\left({ }^{\circ} \mathrm{C}\right)$ \\
Presión de recalentamiento & $2000(\mathrm{kPa})$ \\
Temperatura de recalentamiento & $591,9\left({ }^{\circ} \mathrm{C}\right)$ \\
Presión de proceso industrial & $520(\mathrm{kPa})$ \\
Temp salida proceso industrial & $153\left({ }^{\circ} \mathrm{C}\right)$ \\
Presión de condensador & $10(\mathrm{kPa})$ \\
Poder calorífico inferior del combustible & 45000 \\
Relación de compresión & 10 \\
DTF & 10 \\
\hline
\end{tabular}




\section{METOdologíA Y MÉTOdOS DE ANÁLISIS}

Exergía es la máxima cantidad de trabajo útil que un sistema puede realizar cuando es llevado desde un estado cualquiera hasta las condiciones de referencia ambientales, mediante procesos reversibles [16]. Generalmente la exergía no se conserva, ésta se destruye en el sistema y la destrucción de la exergía es la medida de la irreversibilidad, que es la fuente de pérdida de rendimiento en los sistemas térmicos [9]. El desempeño de los sistemas térmicos usualmente es evaluado por la aplicación de la primera ley de la termodinámica, la cual se basa en el balance de energía. Sin embargo, la primera ley no brinda la información suficiente acerca de la cantidad y el lugar de las irreversibilidades, por lo tanto se debe hacer un estudio en base a la segunda ley para tener en cuenta la calidad de la energía [17].

Para sistemas que operan en régimen permanente como las centrales eléctricas, el balance de masa y análisis de primera ley con cambios de energía cinética y potencial despreciables, se pueden expresar respectivamente como las ecuaciones 1 y 2 , en la ecuación [18].

$$
\begin{aligned}
& \sum_{\text {enrada }} m_{e}=\sum_{\text {salida }} m_{s} \\
& Q_{e}+W_{e}+\sum_{\text {enrada }} m_{c} h_{e}=Q_{s}+W_{s}+\sum_{s \text { salite }} m_{s} h_{s}
\end{aligned}
$$

La segunda ley de la termodinámica se puede expresar como la ecuación 3 :

$$
\sum_{\text {salida }} \dot{m}_{s} S_{s}-\sum_{\text {entrada }} \dot{m}_{e} S_{e} \geq \int \frac{\delta \dot{Q}}{T}
$$

Según [19], el flujo de exergía $\dot{B}$ se puede dividir en distintos componentes: exergía cinética, exergía potencial, exergía física y exergía química, acorde a la ecuación 4 :

$$
\dot{B}=B_{\text {cinetica }}+B_{\text {potencial }}+B_{\text {fisica }}+B_{\text {quimica }}
$$

Los componentes: exergía cinética, exergía potencial y la exergía química no son considerados en este estudio. La ecuación 5 presenta la exergía física específica:

$$
b=\left(h-h_{0}\right)-T_{0}\left(s-s_{0}\right)
$$

Donde $h$ es la entalpia específica, $s$ es la entropía específica y el subíndice $O$ indica el estado muerto restringido (Carranza Sánchez \& de Oliveira, 2015).

El flujo total de exergía física asociado con una corriente de fluido es:

$$
\dot{B}=\dot{m} b=\dot{m}\left(h-h_{0}\right)-T_{0}\left(s-s_{0}\right)
$$

Si se hace un balance de exergía (combinación de la primera y segunda ley de la termodinámica) para un volumen de control, con varias entradas y salidas de flujo, que además genera potencia y recibe de sus alrededores flujo de calor, se tiene la siguiente ecuación (7) (Sanz, 2014).

$\dot{Q}\left(1-\frac{T o}{T}\right)+W_{e}+\sum_{\text {entrada }} \dot{m}_{e} b_{e}=W_{s}+\sum_{\text {salida }} \dot{m}_{s} b_{s}+\dot{B}_{D}$

La variable $B$, es el flujo de exergía en $\mathrm{kW}$, además esta ecuación tiene como objetivo encontrar las pérdidas de energía en los procesos (a esta energía también se le conoce como exergía destruida $B_{D}$.)

La eficiencia de segunda ley o también llamada eficiencia exergética se puede expresar como la relación entre las salidas de exergía útil producida y la consumida, ecuación 8; debido a ésta definición la eficiencia de segunda ley no puede exceder el 100 por ciento.

$$
\varepsilon=\frac{\text { Exergía } \cdot \text { útil } \cdot \text { producida }}{\text { Exergía } \cdot \text { consumida }}
$$

Otro concepto importante al momento de realizar un análisis exergético es la exergía especifica del combustible; según [20] las exergías estándar de la mayoría de los combustibles son similares a sus valores del poder calorífico inferior (PCl) y se pueden calcular con la ecuación 9, expresión utilizada para determinar la eficiencia exergética de la cámara de combustión.

$$
b_{f}=1.06 \times P C I
$$

Donde el PCl es igual al calor liberado por la combustión completa de todos los componentes del combustible y cuando el $\mathrm{H} 2 \mathrm{O}$ en los productos está en forma de vapor. 
Existen herramientas computacionales que permiten dar solución a problemas de ingeniería, una de éstas es el programa EES (Engineering Equation Solver), la función básica de este software es la resolución numérica de ecuaciones algebraicas y diferenciales no lineales. Además, el EES suministra funciones integradas propias y termodinámicas para muchos fluidos [21].

En este trabajo las propiedades termodinámicas de los fluidos, se obtuvieron usando EES. Por medio de las ecuaciones 1 y 2 , se obtienen los flujos de masa para cada una de las extracciones de las turbinas tanto la de baja, como la de alta presión; posteriormente con la ecuación 5 se determina la exergía específica en cada uno de los puntos de la planta térmica, para así proceder a realizar los balances de exergía en los dispositivos encargados del proceso de conversión de energía con la ecuación 7.

Las eficiencias de segunda ley para cada uno de los dispositivos se obtuvieron con la ecuación 8 , teniendo en cuenta que la exergía en la cámara de combustión asociada al combustible se calculó con la ecuación 9.

Los balances exergéticos y las eficiencias de segunda ley para los diferentes componentes, se encuentran en la Tabla 3, donde al ingresar éstas ecuaciones al software EES, éste soluciona las incógnitas y pueden ser presentadas en forma de tablas.

Tabla 3. Balance de exergía y Eficiencia exergética de los componentes

\begin{tabular}{|c|c|c|}
\hline Componente & Balance de Exergía & Eficiencia Exergética \\
\hline C & $B_{1}+W=B_{2}+B_{D}$ & $\varepsilon=\frac{\dot{B}_{2}-\dot{B}_{1}}{W}$ \\
\hline CC & $\dot{B}_{2}+\dot{m}_{f} * b_{f}=\dot{B}_{3}+\dot{B}_{D}$ & $\varepsilon=\frac{B_{3}-B_{2}}{\dot{m}_{f} * b_{f}}$ \\
\hline Cond & $B_{11}=B_{12}+B_{D}$ & ------ \\
\hline HRSG & $B_{4}+\dot{B}_{7}+\dot{B}_{19}=\dot{B}_{5}+\dot{B}_{6}+\dot{B}_{8}+\dot{B}_{D}$ & $\varepsilon=\frac{\dot{B}_{6}+\dot{B}_{8}-\vec{B}_{7}-\dot{B}_{19}}{\dot{B}_{4}-\dot{B}_{5}}$ \\
\hline $\mathrm{IA}$ & $B_{10}+B_{13}+\dot{B}_{18}=\dot{B}_{14}+\dot{B}_{D}$ & $\varepsilon=\frac{\dot{B}_{14}}{\dot{B}_{10}+\dot{B}_{13}+\dot{B}_{18}}$ \\
\hline IC & $\dot{B}_{15}+\dot{B}_{16}=\dot{B}_{17}+\dot{B}_{19}+\dot{B}_{D}$ & $\varepsilon=\frac{\dot{B}_{19}-\dot{B}_{15}}{\dot{B}_{16}-\dot{B}_{17}}$ \\
\hline TG & $\dot{B}_{3}=\dot{B}_{4}+W+\dot{B}_{D}$ & $\varepsilon=\frac{W}{\dot{B}_{3}-\dot{B}_{4}}$ \\
\hline TV & $\begin{array}{l}\dot{B}_{6}+\dot{B}_{8}=B_{7}+\dot{B}_{9}+B_{11}+W+\dot{B}_{D} \\
B_{6}+B_{8}=B_{7}+\dot{B}_{9}+B_{11}+W+B_{D}\end{array}$ & $\varepsilon=\frac{W}{\dot{B}_{6}+\dot{B}_{8}-\dot{B}_{7}-\dot{B}_{9}-\dot{B}_{11}}$ \\
\hline Bomba 1 & $B_{12}+W=\dot{B}_{13}+\dot{B}_{D}$ & $\varepsilon=\frac{B_{13}-B_{12}}{W}$ \\
\hline Bomba 2 & $B_{14}+W=B_{15}+B_{D}$ & $\varepsilon=\frac{B_{15}-B_{14}}{W}$ \\
\hline
\end{tabular}




\section{RESULTADOS Y ANÁLISIS}

El modelo termodinámico del ciclo combinado y la solución de las ecuaciones de balance de masa, energía se realizaron usando EES [22]. La Tabla 4 muestra datos de temperatura, presión, flujo de exergía, flujos másicos para cada estado de la planta.

Tabla 4. Propiedades de los estados en los diferentes puntos correspondientes a la Figura 1

\begin{tabular}{|c|c|c|c|c|c|c|}
\hline Punto & $m[\mathrm{~kg} / \mathrm{s}]$ & $\mathrm{T}\left[{ }^{\circ} \mathrm{C}\right]$ & $P[\mathrm{kPa}]$ & $\mathrm{h}[\mathrm{kJ} / \mathrm{kg}]$ & $s[k J / k W ~ K]$ & $\dot{B}[\mathrm{~kW}]$ \\
\hline 0 & --- & 25 & 101,3 & 298,6 & 5,695 & --- \\
\hline 1 & 300 & 25 & 101,3 & 298,6 & 5,695 & 0 \\
\hline 2 & 300 & 355,2 & 1013 & 637,2 & 5,796 & 92583 \\
\hline 3 & 300 & 1247 & 1013 & 1661 & 6,799 & 309904 \\
\hline 4 & 300 & 616,9 & 101,3 & 922 & 6,835 & 85120 \\
\hline 5 & 300 & 148,2 & 101,3 & 422,9 & 6,044 & 6115 \\
\hline 6 & 31,58 & 591,9 & 6000 & 3639 & 7,145 & 91846 \\
\hline 7 & 31,58 & 429,8 & 2000 & 3313 & 7,222 & 80830 \\
\hline 8 & 27,64 & 591,9 & 2000 & 3672 & 7,682 & 76874 \\
\hline 9 & 25,45 & 401,5 & 520 & 3275 & 7,78 & 59925 \\
\hline 10 & 25,45 & 153 & 520 & 645,3 & 1,873 & 37813 \\
\hline 11 & 2,189 & 58,56 & 10 & 2608 & 8,223 & 3407 \\
\hline 12 & 2,189 & 45,82 & 10 & 191,8 & 0,6493 & 6173 \\
\hline 13 & 2,189 & 45,89 & 520 & 192,6 & 0,6499 & 3059 \\
\hline 14 & 31,58 & 153,3 & 520 & 646,8 & 1,876 & 46940 \\
\hline 15 & 31,58 & 154,6 & 6000 & 655,4 & 1,882 & 47154 \\
\hline 16 & 3,944 & 429,8 & 2000 & 3313 & 7,222 & 10094 \\
\hline 17 & 3,944 & 212,4 & 2000 & 908,7 & 2,447 & 6223 \\
\hline 18 & 3,944 & 153,3 & 520 & 908,7 & 2,49 & 6173 \\
\hline 19 & 31,58 & 222,4 & 6000 & 955,6 & 2,533 & 50509 \\
\hline
\end{tabular}

La Tabla 5 describe las eficiencias exergéticas de los componentes y la eficiencia exergética de la planta; éstas se muestran gráficamente en la Figura 3.

La Figura 4 enseña la distribución de porcentaje del flujo de exergía destruida en cada equipo del sistema. Se encuentra que la cámara de combustión es el equipo que mayor exergía destruye con un $76 \%$, seguido por la caldera de recuperación con un $18 \%$, después le sigue el compresor con un $4 \%$ y por ultimo las turbinas de gas y vapor con un $1 \%$; estos valores guardan la secuencia descrita por [1].
Tabla 5. Eficiencia exergética, flujos de exergía entrada y salida, exergía destruida

\begin{tabular}{ccccc}
\hline Componente & $\varepsilon(\%)$ & $\dot{B_{e}}[\mathrm{~kW}]$ & $\dot{B}_{S}[\mathrm{~kW}]$ & $\dot{B_{D}}[\mathrm{~kW}]$ \\
\hline C & 91,14 & 101579 & 92583 & 8996 \\
CC & 54,7 & 489870 & 309904 & 179981 \\
Cond & 100 & 3407 & 3058 & 348,6 \\
HRSG & 47,31 & 216459 & 174835 & 41624 \\
IA & 99,78 & 47045 & 46940 & 105 \\
IC & 86,68 & 57248 & 56732 & 516 \\
TG & 98,56 & 309904 & 306668 & 3236 \\
TV & 92,59 & 168720 & 166899 & 1821 \\
Bomba 1 & 100 & 3059,611 & 3059 & 0,611 \\
Bomba 2 & 100 & 47210 & 47154 & 56 \\
Planta & 53 & --- & --- & --- \\
\hline
\end{tabular}




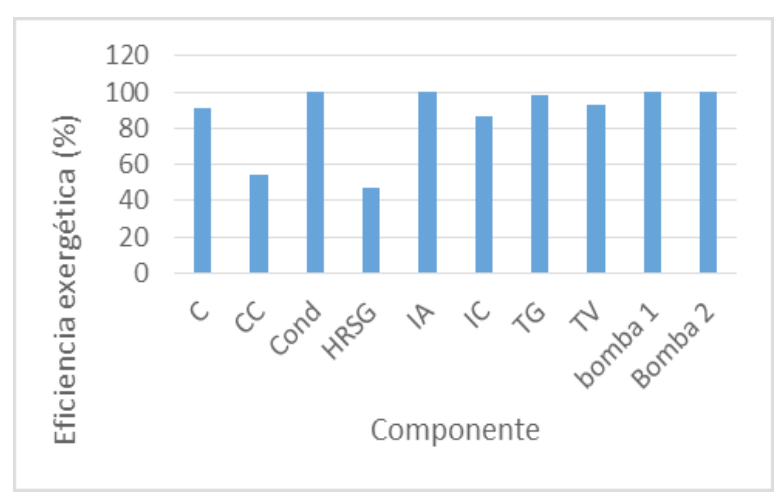

Figura 3. Eficiencia exergética de los componentes de la planta

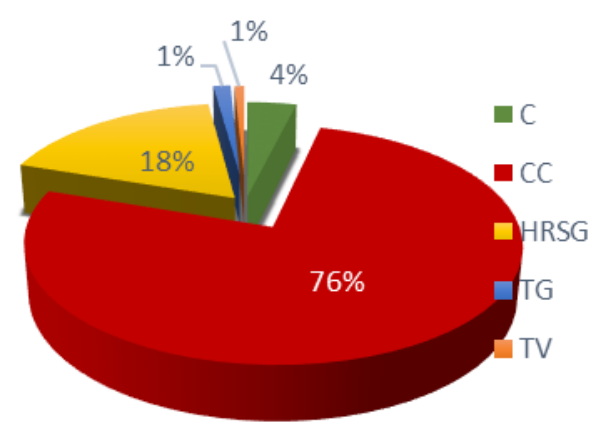

Figura 4. Distribución de porcentajes de la exergía destruida

Con los resultados se verifica que el lugar donde hay mayores pérdidas de exergía es donde se efectúa el proceso de combustión. Según [15], la destrucción de exergía es más alta en la cámara de combustión debido a que las reacciones químicas causan altas irreversibilidades y por la transferencia de calor a través de las grandes diferencias de temperatura entre los gases del quemador y el fluido de trabajo, por lo tanto, uno debería centrarse en la mejora de este dispositivo. El aumento de la proporción de la masa aire-combustible puede causar la disminución de las tasas de destrucción de exergía [15]. Los dispositivos que presentaron la menor pérdida de exergía fueron las bombas y los intercambiadores de calor, estos valores tan bajos se deben principalmente a la baja diferencia de temperaturas entre sus corrientes.

Como se mencionó anteriormente el objetivo de este trabajo es evaluar la disposición de los elementos de la planta propuesta, se tiene que, con las condiciones de operación, la planta presenta una eficiencia exergética del $53 \%$, se puede afirmar que este valor corresponde a un sistema de elevado rendimiento en conversión de energía y por lo tanto que hay una buena distribución de los equipos que conforman la planta y los parámetros de operación de la planta que fueron asumidos hacen que dicha configuración sea altamente eficiente. Si se realiza el cálculo de la eficiencia de primera ley, ésta sería un valor superior a la eficiencia de segunda ley, un rendimiento bruto cercano a las dichas por [5].

\section{CONCLUSIONES}

En este artículo se realizó el análisis exergético de una planta con una distribución particular de sus componentes. Se expusieron de forma concisa los balances de exergía y eficiencia exergética de los dispositivos para conocer los lugares donde se presentan las mayores irreversibilidades. Según el análisis realizado, éste muestra que la disposición de los elementos de la planta es adecuada, debido a que la eficiencia exergética es del $53 \%$ valor que supera incluso a estudios realizado por investigadores como [10].

El análisis exergético revela los componentes que tienen las más altas ineficiencias termodinámicas (destrucción de exergía). La cámara de combustión es el componente principal que contribuye más a la ineficiencia general de la planta. (Aproximadamente el $76 \%$ de la destrucción total de exergía es causada por la CC). Después de la cámara de combustión, el elemento que presenta mayor destrucción de exergía es la HRSG, donde se destruyen 41.6 MW que representan un $18 \%$ de la exergía total que entra al ciclo. El porcentaje de destrucción de exergía en el compresor fue del $4 \%$ mientras que en los demás elementos que conforman la planta fue menos del $2 \%$. Según los resultados mostrados en la Tabla 4, como recomendación la temperatura de los gases que se emiten al ambiente después del paso por la HRSG aún tienen una temperatura elevada que podría ser aprovechada para realizar un posible proceso de calentamiento, logrando con esto un nuevo calor en el ciclo, lo cual aumentaría la eficiencia exergética de la planta.

Es recomendable realizar estudios de viabilidad de la cogeneración y realizar un análisis exergoeconómico de la planta propuesta para determinar el costo de generación de potencia y el costo del vapor de proceso. 


\section{REFERENCIAS}

[1] S. Anvari, S. Jafarmadar, \& S. Khalilarya, "Proposal of a combined heat and power plant hybridized with regeneration organic Rankine cycle: Energy-Exergy evaluation," Energy Conversion and Management, vol. 122, pp. 357-365, August, 2016. DOI: https:// doi.org/10.1016/j.enconman.2016.06.002.

[2] Xiang, W., \& Chen, Y. "Performance improvement of combined cycle power plant based on the optimization of the bottom cycle and heat recuperation," Journal of Thermal Science, vol. $16, \mathrm{n}^{\circ} 1$, pp. 84-89, 2007. Retrieved from: DOI: https://doi. org/10.1007/s11630-007-0084-4.

[3] J.H. Horlock,"Combined power plants - past, present, and future," Journal of Engineering for Gas Turbines and Power, vol. 117, n4, pp. 608-616,October, 1995. Retrieved from: DOI: https://doi. org/10.1115/1.2815448.

[4] C. Casarosa, F. Donatini, \& A. Franco, Thermoeconomic optimization of heat recovery steam generators operating parameters for combined plants. Energy, 29(3), 389-414, 2004. DOI: https://doi. org/10.1016/S0360-5442(02)00078-6.

[5] S. Sabugal García \& F. Gómez Moñux, "Centrales Termicas De Ciclo Combinado: Teoria Y Proyecto," Endesa, Ed. Madrid: España, 2006.

[6] I. Prieto Fernández, Ciclos combinados [online]. Gijón: E.P.S. de Ingeniería, Julio, 2006. Retrieved from: http://ocw.uniovi.es/pluginfile.php/1012/ mod_resource/content/1/1C_C12757_0910/04_ GT14_Centrales_termicas_de_ciclo_combinado. pdf

[7] H.I. Onovwiona, \& V.I. Ugursal, "Residential cogeneration systems: review of the current technology," Renewable and Sustainable Energy Reviews, vol. 10, n 5, pp. 389-431, 2006. Retrieved from: Doi: http://doi.org/10.1016/j.rser.2004.07.005

[8] Y.M. El-Sayed, \& R.A. Gaggioli, "A Critical Review of Second Law Costing Methods-I: Background and Algebraic Procedures," Journal of Energy Resources Technology, vol. 111, $\mathrm{n}^{\circ} 1$, pp. 1-7, Mar. 1989. Retrieved from: DOI: https://doi. org/10.1115/1.3231396.

[9] I.H. Aljundi, "Energy and exergy analysis of a steam power plant in Jordan". Applied Thermal Engineering, vol. 29, $\mathrm{n}^{\circ} 2-3$, pp. 324-328, February 2009. Retrieved from: DOI: https://doi.org/10.1016/j. applthermaleng.2008.02.029.
[10] E. Ersayin, \& L. Ozgener, "Performance analysis of combined cycle power plants: A case study," Renewable and Sustainable Energy Reviews, vol. 43, pp. 832--842, 2015. Retrieved from: DOI: https:// doi.org/10.1016/j.rser.2014.11.082.

[11] F. Petrakopoulou, G.Tsatsaronis, T. Morosuk, \& A. Carassai, "Conventional and advanced exergetic analyses applied to a combined cycle power plant," Energy, vol. 41, n 1, pp. 146-152, 2012. Retrieved from: Doi: http://doi.org/10.1016/j.energy.2011.05.028

[12] A.M. Bassily, "Modeling, analysis, and modifications of different GT cooling techniques for modern commercial combined cycle power plants with reducing the irreversibility of the HRSG," Applied Thermal Engineering, vol $53 \mathrm{n}^{\circ} 1$, pp. 131146, April, 2013. DOI: https://doi.org/10.1016/j. applthermaleng.2013.01.002.

[13] E.V. Torres Gonzáles, M. Salazar Peryra, R. Lugo Leyte, \& A. Torres, "Analisis exergoeconómico de una planta de cogeneración con turbina de gas considerando el proceso de formación del residuo,"CIBIM 10, Oporto,Portugal,2011-CIBEM 10, Porto, Portugal,2011, pp. 1387-1392, Mar. 2016. Retrieved from: https://www.researchgate.net/ publication/279657120_Analisis_exergoeconomico_de_una_planta_de_cogeneracion_con_turbina_de_gas_considerando_el_proceso_de_formacion_del_residuo

[14] Sanjay, \& B.N. Prasad, "Energy and exergy analysis of intercooled combustion-turbine based combined cycle power plant". Energy, vol. 59, pp. 277-284, 2013. Retrieved from: DOI: https://doi. org/10.1016/j.energy.2013.06.051.

[15] E. Açikkalp, H. Aras, \& A. Hepbasli, "Advanced exergy analysis of an electricity-generating facility using natural gas," Energy Conversion and Management, vol. 82, pp. 146-153, 2014. Retrieved from: DOI: https://doi.org/10.1016/j.enconman.2014.03.006.

[16] D. Sanz Amaya, "Análisis y Optimización exergética de una planta de Cogeneración para la Industria Azucarera,"Tesis maestria, Facultad Ingeniería Mecánica, Universidad Pedagógica de Pereira, Pereira, 2014.

[17] A.M. Elsafi, "Exergy and exergoeconomic analysis of sustainable direct steam generation solar power plants," Energy Conversion and Management, vol.103, pp. 338-347, October, 2015 DOI: https:// doi.org/10.1016/j.enconman.2015.06.066.

[18] Y. A., Cengel, \& M.A. Boles, Termodinamica, 7th. ed. México D.F.: McGraw-Hill Companies. Inc.

Rev. I²+D. Vol.17 (1). Enero-Junio. 2017, pp. 49-58. ISSN 1900-771X e-ISSN 2422-4324 
2011, pp. 1456. Disponible: http://es.slideshare.net/nomarzedlav/termodinamica-7ma-edicin-yunus-a-cengel-michael-a-boles

[19] Y.A. Carranza Sánchez, \& S. de Oliveira Jr., "Exergy analysis of offshore primary petroleum processing plant with CO2 capture," Energy, vol. 88, pp. 4656, August, 2015. DOI: https://doi.org/10.1016/j. energy.2015.05.130.

[20] I. Dincer, \& M.A. Rosen, "Chapter 12 - Exergy Analysis of Steam Power Plants,". In Exergy, 2th. ed. Elsevier, 2013, pp. 261-284. DOI: https://doi. org/10.1016/B978-0-08-097089-9.00012-7.

[21] Y.A. Cengel, \& A.J. Ghajar, Transferencia de calor y masa, Fundamentos y aplicaciones, 4th. ed. Ciudad de Mexico: McGrawwHill, 2012.

[22] N. Fonseca, Engineering Equation Solver (EES, Facultad de Ingeniería Mecanica, Universidad Tecnológica de Pereira, Pereira, 2016. Retrieved from: http://www.academia.edu/9414760/Engineering_ equation_solver_EES 